\title{
Uso y abuso del celular por parte de estudiantes de la Universidad Santo Tomás, una perspectiva estadística
}

\section{Cell phone use and abuse by students of Santo Tomás University, a statistical perspective}

Julian Enrique Castellanos Rosas juliancastellanos@usantotomas.edu.co
Luz Adriana Rodriguez Padilla

luzrodriguezp@usantotomas.edu.co

Cristian Fernando Téllez Piñerezc

cristiantellez@usantotomas.edu.co

\begin{abstract}
Resumen
¿Considera que invierte demasiado tiempo en su celular? Para esta generación el dispositivo móvil se ha convertido en un dispositivo indispensable en la vida cotidiana. El internet es el motivo principal de la globalización, pues estos dispositivos reúnen todas las mejores características de las nuevas tecnologías de innovación e información en un solo objeto, volviendo al dispositivo móvil el artefacto más popular y usado por las personas en todo el mundo. Esto ha producido enormes y evidentes cambios en las sociedades y personas, por este motivo, se decidió hacer un estudio en el cual, se caracterizó el uso del celular enfocándose en los estudiantes de la Universidad Santo Tomas. Este estudio se realizó utilizando técnicas de muestreo para recolectar la información y posteriores estimaciones y técnicas de análisis multivariado para generar la validación de la encuesta y los indicadores. Los principales resultados que se encontraron fueron indicios de adicción manifestada en angustia al no hacer uso de su celular por tiempos prolongados, así como también beneficios académicos bajo un uso correcto del dispositivo.
\end{abstract}

Palabras clave: Análisis multivariado, Celular, Dependencia, M-learning, Muestreo.

\footnotetext{
aEstudiante Facultad de Estadística Universidad Santo Tomás

b Estudiante Facultad de Estadística Universidad Santo Tomás

${ }^{\mathrm{c}} \mathrm{Ph} . \mathrm{D}(\mathrm{c})$ Ciencias Estadística. Docente Universidad Santo Tomás
} 


\begin{abstract}
Do you think you spend too much time on your cell phone? For this generation, this device has become indispensable in everyday life. The internet is the main reason for globalization, and these devices, all together contain the best features of new innovations and information technologies in a single object, making the cell phone the most popular device used by people around the world. This has produced enormous and evident changes in societies and people, for this reason, a study was done in which the use of cell phones was analyzed, focusing on the students of the Santo Tomas University. This study was carried out using sampling techniques to collect the information and subsequent estimates and multivariate analysis techniques to generate the validation of the survey and the indicators. The main results that were found were indications of addiction, which was manifested in anguish when not using their cell phone for long periods of time, as well as academic benefits under correct use of the device.
\end{abstract}

Keywords: Cell phone, Dependency, M-learning, Multivariate Analysis, Sampling.

\title{
1. Introducción
}

Hoy en día el uso del celular no solo en los jóvenes, sino, en la población en general se ha establecido como una necesidad, ya que, por medio de este dispositivo se agenda reuniones, se facilita la comunicación con personas cercanas o con compañeros de trabajo y también se crea vida social. Si bien, el primer celular se introdujo en Estados Unidos por Martin Cooper en 1973 y la revolución más grande se tuvo en el 2002 gracias a la tercera generación de telefonías móviles que permitía, no solo el manejo de voz, sino, el tener acceso a internet desde el dispositivo (Agar 2013). Desde entonces, ¿las personas han tomado conciencia en la forma en que invierten su tiempo al hacer uso del dispositivo?

Para el caso de Colombia, no contó con este inicio de revolución, sino, hasta 1994 con operadores como Celumóvil o Comcel, manteniendo tarifas elevadas y poco accesibles al público. No fue sino hasta el 2006, con la llegada al país de telefonías como, Movistar y Tigo (Cáceres 2018) que se logra la adquisición de dispositivos móviles para la mayoría de las familias, dado que los precios de los celulares y las tarifas de los planes telefónicos que ofrecían eran asequibles.

A partir de este año, el celular formó parte de la vida cotidiana de una generación, que a la fecha tiene entre los 16 y 35 años. Tomando como base lo anterior y que actualmente las sociedades se desenvuelven conforme la tecnología avanza y se desarrolla, más exactamente, las tecnologías de la información y comunicación (TIC), producen notorios cambios e influencias sociales que se evidencian en las nuevas formas de vida y comportamientos en los jóvenes, pues, el dispositivo móvil se ha convertido es una fuente de identidad donde pueden expresar libremente sus ideas y opiniones en Colombia. 
Por otro lado, el DANE registró que, en 2016, en el 96,4\% de los hogares del país algún miembro contaba con teléfono celular (DANE 2017). Es decir, es innegable el hecho de la necesidad que se ha creado hacia este dispositivo, siendo escasas las personas que no usan ningún dispositivo, las razones son innumerables: redes sociales y millones de apps que nos han hecho la vida más fácil y confortable con sus diferentes funciones que van desde lo complejo como un asistente virtual hasta lo más simple como una alarma, esto ha transformado la manera en que nos relacionamos con el mundo al generarse cambios en las personas paralelamente a la evolución de los teléfonos móviles. Se ha llevado tan lejos el uso de esta tecnología que ya nos resulta esencial para la mayoría de las actividades que realizamos día a día he incluso son capaces de imponer modas e influencias sociales.

A raíz de esta problemática, se han originado estudios respecto al uso, dependencia e influencia que marcan estos dispositivos en la vida de las personas. Dentro de estos estudios se encuentra una investigación realizada por Arias (2011). Siendo el tema central la influencia de los smartphones (celulares) sobre los estilos de vida de los jóvenes universitarios y ejecutivos en la ciudad de Bogotá, concluyendo que la influencia de los teléfonos inteligentes en los estilos de vida de los jóvenes universitarios, ha marcado gran impacto, pues han llegado a crear dependencia hacia esta tecnología a través de la creación de diferentes aplicaciones, pasando a ser en algunos usuarios adicción. Su investigación indicó que algunos jóvenes se les hace imposible desconectarse de su celular, creando problemas de aislamiento.

Por suparte, Estupiñan (2008) trabajó acerca de la influencia del teléfono móvil en los cambios culturales y sociales de los jóvenes universitarios de dicha universidad, se encontró que estos jóvenes ven en el celular, un símbolo que los diferencia del resto, ellos dicen que esto es fundamental en casos de interacción social como la amistad, la conquista o evolución de pareja y la evolución personal, entre otras, que dejan ver que el joven más allá de encontrar en el celular una herramienta de suplir necesidades únicas de comunicación, también encuentra necesidades sociales y personales como el estatus social y la imagen personal. Un tercer trabajo encontrado fue el realizado por Organista-Sandoval, Serrano-Santoyo, McAnally-Salas y Lavigne (2013), donde el objetivo era percibir la existencia de usos educativos que puedan efectuarse con el dispositivo móvil como también la adaptación a la tecnología tanto de estudiantes como de maestros. Para determinar esto, el diseño de muestreo utilizado es no probabilístico, lo que conlleva a que los estadísticos que usaron fueron de carácter descriptivo.

Al no contar con suficientes estudios para medir la adicción a un dispositivo móvil, se presenta el fenómeno en el cual, medios de comunicación y la sociedad estigmatizan conductas de usuarios como inapropiadas, sin reconocer el avance desproporcional en tan poco tiempo de esta tecnología, lo que conlleva a que los jóvenes se adapten al uso del mismo desde su visión prematura de la vida, sin personalidad o madurez suficiente, cosas con las que la sociedad tampoco cuenta para su manejo. Esta forzada adaptación de jóvenes a los dispositivos móviles, genera una alerta para la comunidad científica, siendo el eje para investigaciones y es necesario caracterizar el uso de los celulares. De esta manera y desde los inicios del 
dispositivo móvil se logra identificar que proporciones de la población usuaria, tienen realmente problemas de inseguridad, irritación y aislamiento, causadas por el socializar o no con su celular (Graner et al. 2007), incluso al caracterizar el uso del mismo se tendrá noción de la forma como invierten el tiempo y en que aplicaciones lo hacen, identificando si han dejado de ser situaciones cotidianas: el realizar una compra, el uso en la academia para una comunicación masiva más efectiva o el generar actividades en línea para un mayor aprendizaje y se han convertido en un problema de compras excesivas por el gran mercado disponible y la facilidad de acceder a él o una distracción en la actividad académica.

Como tampoco se puede dejar de lado el hecho de que ahora mismo para garantizar una buena calidad de educación es necesario el uso de esta tecnología, que no solo nos facilita el acceso a una cantidad inmensurable de información sino que también hace posible la creación de espacios, en los cuales, se crean oportunidades de aprendizaje incluyentes y capaces de sincronizarnos ante un mundo actual que se ha tecnificado y así aprovechar estos nuevos recursos, potenciando el desarrollo de nuevas áreas de conocimiento que nos permite ir a la vanguardia de una globalización inminente, siendo el dispositivo móvil una herramienta fundamental dada su popularidad. Este fenómeno es también llamado M-learning (aprendizaje móvil), una rama del E-learning o aprendizaje electrónico, el cual nos proponen un aprendizaje interactivo a través de diferentes dispositivos móviles y de manera virtual lo cual trae consigo diferentes beneficios ya que la persona tiene total manejo del tiempo y del lugar en el que desee adquirir los conocimiento, lo cual agiliza su proceso de formación, así se menciona en el estudio realizado por Gómez-Ramirez, Valencia-Arias y Duque (2019) en cual indican que el M-learning tiene un enorme potencial y promete nuevas oportunidades que los métodos tradicionales no pueden ofrecer.

Teniendo en cuenta todo lo anterior y la importancia que puede tener para la Universidad Santo Tomás contar con un estudio de corte probabilístico que caracterice el uso del celular en sus estudiantes, se decidió hacer este artículo, estudiando ese fenómeno en particular. Cabe resaltar que este trabajo es un primer acercamiento a medir el fenómeno del uso del celular en estudiantes el cual puede servir de pilar para un estudio más ambicioso, por ejemplo, realizarlo a nivel de todas las sedes de la Universidad o realizarlo a nivel de todas las universidades del país. 


\section{Diseño de muestreo y recolección de la informa- ción}

A continuación, se mostrará la definición y la metodología para la realización de un diseño de muestreo sistemático, con sus respectivas probabilidades de inclusión y estimadores, así como también se describirá el trabajo de campo realizado para este estudio.

\subsection{Diseño de muestreo}

El diseño de muestreo planteado para llevar a cabo esta investigación fue el diseño Sistemático, el cual, es usado frecuentemente por su sencillez y buenos resultados cuando no se cuenta con un marco de muestreo explícito (Särndal, Swensson y Wretman, 1992) .

Como se mencionó anteriormente, este diseño no requiere de un marco de muestreo explícito, y es aplicable cuando los elementos de la población sobre la que se realiza el estudio se pueden ordenar de alguna forma, por ejemplo, orden de llegada a la universidad, entre otras (Gutiérrez 2016). Este procedimiento de muestreo se basa en tomar muestras de una manera directa y ordenada a partir de una regla determinística, también llamada sistemática y cuyo algoritmo de selección es el siguiente:

1. Asumir un ordenamiento de los individuos de la población

2. Definir el tamaño del salto, que se denota con la letra $a$. Este salto usualmente se calcula por $a=\left[\left|\frac{N}{n}\right|\right]$ En donde $\left[\left|\frac{N}{n}\right|\right]$ representa la parte entera del cociente $N / a, N$ es el tamaño de la población y $n$ es el tamaño de muestra..

3. Se selecciona un primer individuo en la población (primera unidad muestral) de forma aleatoria con probabilidad $\frac{1}{a}$, un entero $r$ tal que $1 \leqslant r \leqslant a$.

4. Luego de esta primera unidad, se elegirá la segunda después de dar un salto de $a$ unidades y así sucesivamente se seleccionarán el resto de individuos para la muestra.

\subsection{Estimadores}

Las probabilidades de inclusión de primer y segundo orden para este diseño muestral están dadas por las ecuaciones 1 y 2 respectivamente:

$$
\begin{gathered}
\pi_{k}=\frac{1}{a} \\
\pi_{k l}=\left\{\begin{array}{cc}
\frac{1}{a} & \forall k, l \in S \\
0 & \text { e.o.c. }
\end{array}\right.
\end{gathered}
$$




\section{Julian Enrique Castellanos Rosas, Luz Adriana Rodriguez Padilla \& Cristian Fernando Téllez Piñerez}

Una vez que el diseño de muestreo es definido, la estrategia se completa con el uso del estimador de Horvitz-Thompson, por ser este un diseño sin reemplazo (Gutiérrez 2016). Para este estudio en particular, se analizaron variables de tipos cuantitativas, como la edad y cualitativas, como marcas de los celulares.El estimador del total junto con su varianza están dadas por las ecuaciones 3 y 4 respectivamente.

$$
\begin{gathered}
\widehat{t}_{\pi}=\sum_{S r} \frac{y k}{\pi_{k}}=a t_{s r} \quad, \quad t_{s r}=\sum_{k \epsilon S_{r}} y_{k} \\
\operatorname{Var}_{S I S}\left(\widehat{t}_{\pi}\right)=a \sum_{r=1}^{a}\left(t_{s r}-\bar{t}\right)^{2} \quad, \quad \bar{t}=\sum_{r=1}^{a} \frac{t_{s r}}{a}=\frac{t}{a}
\end{gathered}
$$

Para este diseño no existe el estimador de la varianza, ya que algunas probabilidades de inclusión de segundo orden son nulas. Para solventar esto, en la literatura especializada existen varias opciones, entre ellas, la estimación Bootstrap (Tellez et al. 2014) y utilizar el estimador de la varianza del muestreo aleatorio simple como lo recomienda Särndal et al. (1992). Para este ejercicio se siguieron los lineamientos de Särndal et al. (1992) dado que los resultados eran muy similares.

Por otro lado, para la estimación de los parámetros que conlleve las variables cualitativas se estimarán proporciones y sus varianzas, las cuales están definidas en las ecuaciones 5 y 6 respectivamente.

$$
\widehat{\rho}=\frac{\widehat{t}_{\pi}}{\hat{t}_{z_{k}}}=\frac{\widehat{t}_{\pi}}{\widehat{N}}=\frac{a \sum_{S} y_{k}}{\sum_{S} \frac{1}{\frac{1}{a}}}=\frac{\frac{N}{n} \sum_{S} y_{k}}{N}=\frac{\sum_{S} y_{k}}{n} \quad, \quad z_{k}=1 \quad \forall k \in S
$$

donde

$$
y_{k}=\left\{\begin{array}{lc}
1 & \text { si } k \text { cumple con la característica de interés } \\
0 & \text { e.o.c. }
\end{array}\right.
$$

Varianza estimada del estimador:

$$
\widehat{\operatorname{Var}}(\widehat{\rho})=\frac{N^{2}}{n}\left(1-\frac{n}{N}\right) \frac{\widehat{\rho}(1-\widehat{\rho})}{n}
$$

Por otro lado, para obtener estimaciones con márgenes de errores $(e)$ inferiores a $3.5 \%$, una confianza del $95 \%(1-\alpha)$ y una prevalencia $(P)$ inferior al $25 \%$ se obtuvo un tamaño de muestra mínimo de 559 estudiantes. Este tamaño de muestra va a expandir a la población estudiantil de 11102 alumnos, de la Universidad Santo Tomás, en el periodo 2018-2. Para calcular el tamaño de muestra se utilizó la ecuación 7 la cual fue propuesta por Kish (1965).

$$
n=\frac{z_{1-\frac{\alpha}{2}}^{2} P(1-P) N}{e^{2} N+z_{1-\frac{\alpha}{2}}^{2} P(1-P)}
$$




\subsection{Trabajo de campo}

Siguiendo el algoritmo de selección mencionado anteriormente para un diseño sistemático, se realizó la recolección de la información con un equipo de 23 personas, los cuales conformaban la totalidad de los estudiantes del curso de Muestreo probabilístico. La información se recolectaba por medio de los celulares en un formulario digital.

$\mathrm{Al}$ ser un diseño sistemático la ausencia de participación se controló tomando al siguiente estudiante de acuerdo al salto y no se tuvo participación parcial, por lo tanto no hubo tratamientos de no respuesta.

Los integrantes del equipo se distribuyeron en puntos estratégicos de la universidad, donde había afluencia de estudiantes, en las jornadas mañana, tarde y noche durante una semana (lunes a sábado) con el fin de poder realizar los saltos sistemáticos propios del diseño y absorber la mayor variabilidad posible en la información recolectada. Ahora bien, para poder cumplir con el tamaño mínimo propuesto se decidió que cada encuestador realizara mínimo 27 encuestas en los distintos puntos seleccionados. Algunos de los puntos fueron: Entrada, salida y cafeterías de la universidad.

Por otro lado, en términos del cuestionario, este constaba de 28 preguntas divididas en 4 aspectos a evaluar. Dichos aspectos son: Información general, información del dispositivo, influencia del dispositivo móvil en la vida diaria y dependencia al dispositivo. Se ajustó el cuestionario siguiendo la investigación realizada por Young (1998), dado que el objetivo es caracterizar el uso de una nueva tecnología. Antes de aplicar el cuestionario, se realizó una validación de unidimensionalidad para cada uno de los 4 aspectos de manera gráfica (Muñiz 2005), cabe resaltar que cada pregunta es representada por un vector en el gráfico como se muestra a continuación:

En la Tabla y Figura 1 se observa que las preguntas 1, 2 y 5 son las que más contribuyen información en la componente 1 con $22.9 \%, 36.9 \%$ y $32.6 \%$ respectivamente. Sin embargo, las preguntas 3,4 y 6 , si bien, no contribuyen en la misma cantidad que las anteriores, la información que brindan en la componente es relevante desde el punto de vista psicológico para poder medir de manera asertiva la componente. 


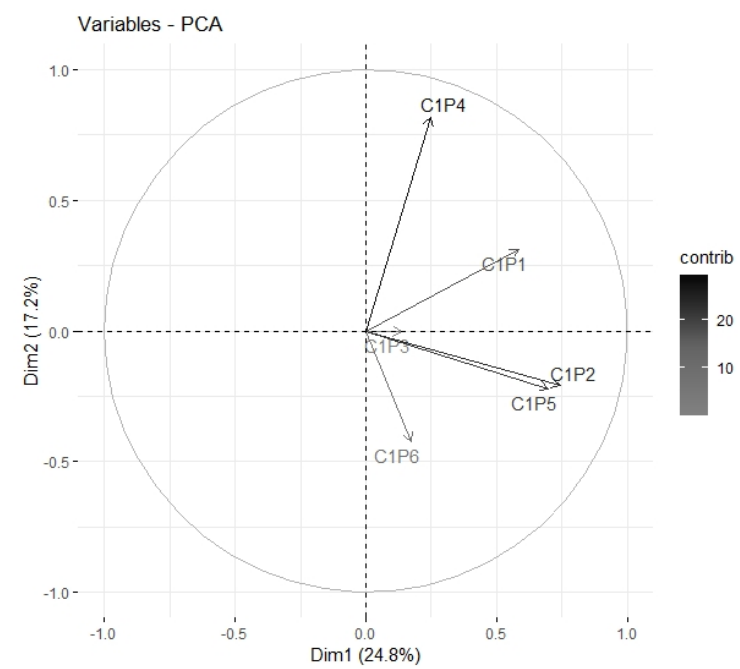

Figura 1: Información personal

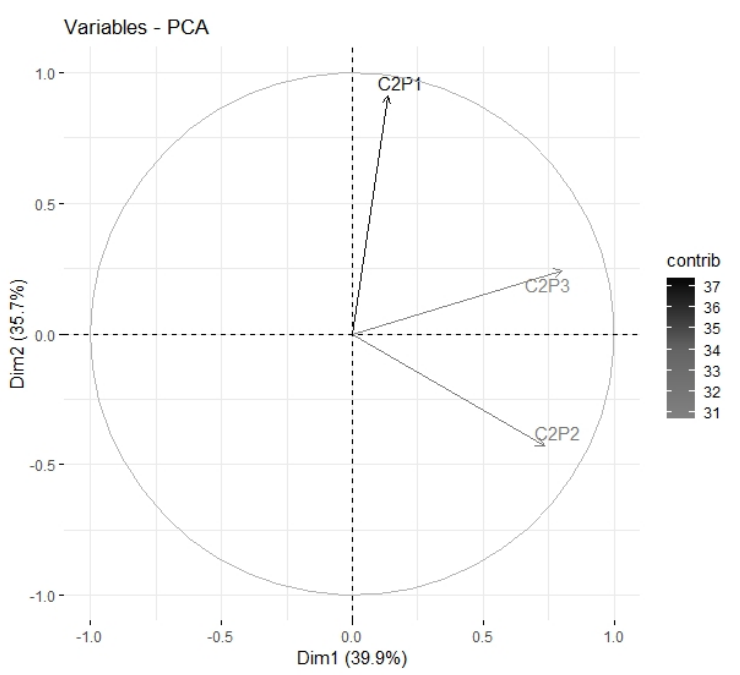

Figura 2: Información del dispositivo móvil

Tabla 1: Contribuciones Categoría 1

\begin{tabular}{|ll|}
\hline & Dim.1 \\
\hline C1P1 & 22.9 \\
C1P2 & 36.9 \\
C1P3 & 1.2 \\
C1P4 & 4.1 \\
C1P5 & 32.6 \\
C1P6 & 2.1 \\
\hline
\end{tabular}

Tabla 2: Contribuciones Categoría 2

\begin{tabular}{|ll|}
\hline & Dim.1 \\
\hline C2P1 & 1.4 \\
C2P2 & 44.9 \\
C2P3 & 53.1 \\
\hline
\end{tabular}

Fuente: Elaboración propia.

Fuente: Elaboración propia.

Este mismo fenómeno se presenta en las Tablas y Figuras 2 donde las preguntas 2 y 3 son las que mayor información aportan a la componente sin dejar a un lado la importancia de la pregunta 3 . Un caso similar sucede en las componentes de las Tablas y Figuras 3 y 4. 


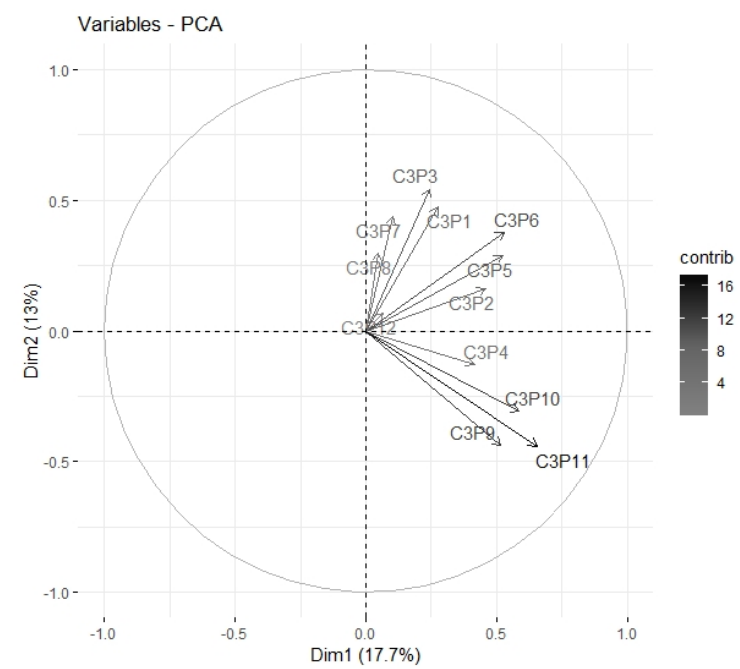

Figura 3: Influencia del dispositivo móvil en la vida diaria

Tabla 3: Contribuciones Categoría 3

\begin{tabular}{|ll|}
\hline & Dim.1 \\
\hline C3P1 & 3.5 \\
C3P2 & 9.8 \\
C3P3 & 2.7 \\
C3P4 & 8.08 \\
C3P5 & 12.8 \\
C3P6 & 13.1 \\
C3P7 & 0.4 \\
C3P8 & 0.1 \\
C3P9 & 12.5 \\
C3P10 & 16.1 \\
C3P11 & 20.2 \\
C3P12 & 0.2 \\
\hline
\end{tabular}

Fuente: Elaboración propia.

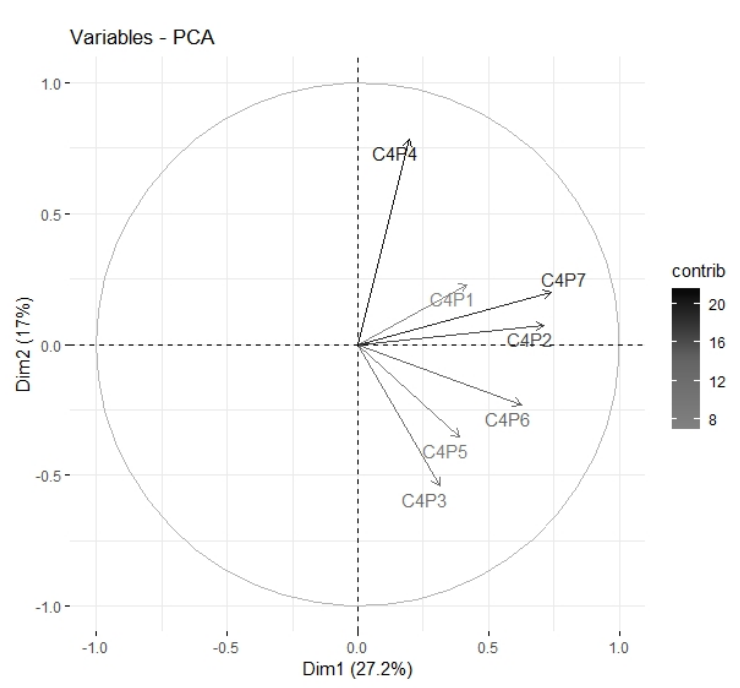

Figura 4: Dependencia al dispositivo

Tabla 4: Contribuciones Categoría 4

\begin{tabular}{|ll|}
\hline & Dim.1 \\
\hline C4P1 & 9.1 \\
C4P2 & 26.7 \\
C4P3 & 5.08 \\
C4P4 & 2.00 \\
C4P5 & 7.8 \\
C4P6 & 20.5 \\
C4P7 & 28.6 \\
\hline
\end{tabular}

Fuente: Elaboración propia.

Por otro lado, en términos de validación interna del constructo, se calcularon los alpha de Cronbach para cada componente, reportando un alpha promedio alrededor del $50 \%$. Si se toma como referencia que los módulos no adoptados que el Icfes aplica en las pruebas Saber Pro tienen, inclusive, alfas de Cronbach inferiores al $40 \%$, podemos concluir que el constructo, en términos de dimensionalidad y validación interna, está validado. 
Una vez validado este cuestionario en términos de unidimensionalidad, se procede aplicar a los estudiantes de la Universidad Santo Tomás de manera aleatoria como se mostró anteriormente con el fin, de poder realizar inferencias válidas de los resultados a toda la universidad.

\section{Resultados}

Con base en la muestra recolectada se puede observar que, en promedio, los estudiantes de la Universidad Santo Tomás tiene 21 años. Si bien, en Colombia, la población de mujeres es mayor que la de los hombres, en la Universidad Santo Tomás, hay un equilibrio en esta variable. Se pudo estimar que, el $51 \%$ de los estudiantes son hombres y $49 \%$ son mujeres, como se puede observar en la Tabla 3. Lo anterior es un buen indicio para la muestra ya que está expandiendo a la población real de estudiantes de la Universidad.

\begin{tabular}{|c|c|c|c|c|c|c|c|c|c|}
\hline & \multicolumn{2}{|c|}{ Adicción al celular } & \multicolumn{2}{|c|}{$\begin{array}{l}\text { Accidente por } \\
\text { uso celular }\end{array}$} & \multicolumn{5}{|c|}{$\begin{array}{l}\text { Angustia al no } \\
\text { usar el celular }\end{array}$} \\
\hline Edad & Femenino & Masculino & No & $\mathrm{Si}$ & \multicolumn{2}{|c|}{ No } & \multicolumn{3}{|c|}{$\mathbf{S i}$} \\
\hline$[16-18)$ & $4,14 \%$ & $1,08 \%$ & $0,72 \%$ & $0,90 \%$ & \multicolumn{2}{|c|}{$3,06 \%$} & \multicolumn{3}{|c|}{$2,16 \%$} \\
\hline$[18-21)$ & $26,66 \%$ & $21,08 \%$ & $23,96 \%$ & $13,51 \%$ & \multicolumn{2}{|c|}{$29,18 \%$} & \multicolumn{3}{|c|}{$18,56 \%$} \\
\hline$[21-24)$ & $19,64 \%$ & $14,59 \%$ & $33,51 \%$ & $10,45 \%$ & \multicolumn{2}{|c|}{$21,62 \%$} & \multicolumn{3}{|c|}{$12,61 \%$} \\
\hline$[24-27)$ & $3,96 \%$ & $3,60 \%$ & $8,11 \%$ & $2,16 \%$ & \multicolumn{2}{|c|}{$4,50 \%$} & \multicolumn{3}{|c|}{$3,06 \%$} \\
\hline$[27-30)$ & $1,62 \%$ & $0,90 \%$ & $3,24 \%$ & $0,36 \%$ & \multicolumn{2}{|c|}{$1,44 \%$} & \multicolumn{3}{|c|}{$1,08 \%$} \\
\hline$[30-33)$ & $1,08 \%$ & $0,90 \%$ & $1,26 \%$ & $0,72 \%$ & \multicolumn{2}{|c|}{$1,26 \%$} & \multicolumn{3}{|c|}{$0,72 \%$} \\
\hline$[33-36)$ & $0,72 \%$ & $0,54 \%$ & $0,72 \%$ & $0,54 \%$ & \multicolumn{2}{|c|}{$0,36 \%$} & \multicolumn{3}{|c|}{$0,90 \%$} \\
\hline$[36-41]$ & $0,18 \%$ & $0,36 \%$ & $0,90 \%$ & $0,00 \%$ & \multicolumn{2}{|c|}{$0,36 \%$} & \multicolumn{3}{|c|}{$0,18 \%$} \\
\hline \multirow[t]{2}{*}{ Total } & $58,01 \%$ & $43,06 \%$ & $72,42 \%$ & $28,64 \%$ & $61,7 \mathrm{~s}$ & & & $27 \%$ & \\
\hline & \multicolumn{2}{|c|}{ Sexo } & \multicolumn{2}{|c|}{ Conexión internet } & \multicolumn{5}{|c|}{ Estrato } \\
\hline Edad & Femenino & Masculino & Datos & Wifi & 1 & 2 & 3 & 4 & 5 \\
\hline$[16-18)$ & $2,52 \%$ & $2,70 \%$ & $1,98 \%$ & $3,24 \%$ & $0 \%$ & $1 \%$ & $3 \%$ & $2 \%$ & $0 \%$ \\
\hline$[18-21)$ & $26,31 \%$ & $20,36 \%$ & $21,80 \%$ & $25,94 \%$ & $1 \%$ & $10 \%$ & $27 \%$ & $9 \%$ & $1 \%$ \\
\hline$[21-24)$ & $15,49 \%$ & $18,74 \%$ & $15,13 \%$ & $19,10 \%$ & $1 \%$ & $4 \%$ & $20 \%$ & $7 \%$ & $1 \%$ \\
\hline$[24-27)$ & $2,88 \%$ & $4,68 \%$ & $4,14 \%$ & $3,42 \%$ & $0 \%$ & $2 \%$ & $4 \%$ & $2 \%$ & $0 \%$ \\
\hline$[27-30)$ & $1,08 \%$ & $1,44 \%$ & $1,44 \%$ & $1,08 \%$ & $0 \%$ & $1 \%$ & $1 \%$ & $0 \%$ & $0 \%$ \\
\hline$[30-33)$ & $0,54 \%$ & $1,44 \%$ & $1,44 \%$ & $0,54 \%$ & $0 \%$ & $1 \%$ & $1 \%$ & $0 \%$ & $0 \%$ \\
\hline$[33-36)$ & $0,36 \%$ & $0,90 \%$ & $1,08 \%$ & $0,18 \%$ & $0 \%$ & $0 \%$ & $1 \%$ & $0 \%$ & $0 \%$ \\
\hline [36-41] & $0,00 \%$ & $0,54 \%$ & $0,36 \%$ & $0,18 \%$ & $0 \%$ & $0 \%$ & $1 \%$ & $0 \%$ & $0 \%$ \\
\hline Total & $49,20 \%$ & $50,80 \%$ & $47,38 \%$ & $53,68 \%$ & $2 \%$ & $19 \%$ & $57 \%$ & $20 \%$ & $3 \%$ \\
\hline
\end{tabular}

Por otro lado, se logró estimar que el $75 \%$ de los estudiantes de la universidad tuvieron su primer celular antes de los 14 años, lo cual, contrastado con la edad actual, se tiene un margen aproximado de 7 años de uso del celular, lo que conlleva a pensar que, tener y usar el celular a diario es un hábito para los jóvenes. En este sentido, Bringué y Sádaba-Chalezquer (2011) confirma que esta situación se percibe gracias a la expansión del dispositivo móvil y uso a temprana edad. El inicio temprano del uso de estas tecnologías está propiciando la adopción de nuevas pautas de conducta y afectando a sus relaciones personales y familiares (De Morentin et al. 2014), como también, se señala el aumento de la vulnerabilidad al desarrollo de adicciones, tanto de carácter comportamental como de consumo de sustancias tóxicas, además de la disminución del contacto presencial en favor 
de la interacción virtual (Muñoz-Miralles et al. 2016).

Como se mencionó anteriormente, muchas preguntas surgen con la aparición y excesivo uso del celular, tanto por los jóvenes, como por los adultos. Una de las preguntas más usuales que se hace hoy en día y la cual, se indagó en los estudiantes de la universidad es: ¿Todos los jóvenes tienen un celular inteligente (smartphone)? Con base en la información recolectada y aunque parezca algo contradictorio, pertenecer a un estrato bajo o no contar con empleo, no es un impedimento para la adquisición de un celular inteligente, pues los datos recogidos en la Universidad Santo Tomas muestran que el $77.6 \%$ de los estudiantes de la universidad son de estratos 1,2 y 3 , de los cuales el $50.2 \%$ no trabaja, sin embargo, se estimó que el $98 \%$ de las estudiantes poseen un Smartphone. Esto puede deberse según Rodríguez, Restrepo y García (2017) a la reducción en su costo y su utilidad como herramienta para el trabajo, el estudio y la diversión, lo cual ha permitido su acceso a la población en general, independientemente de su edad y nivel socioeconómico.

Pero más allá de saber si poseen o no un dispositivo móvil, se quiere caracterizar el uso de este por los estudiantes. Logrando observar que el $58.9 \%$ considera importante el uso del dispositivo móvil para el desarrollo de la clase, pero ¿traerá esto beneficios académicos? Uno de los resultados más interesantes es el uso del dispositivo móvil para plagio donde se encontró que, con una confianza del $95 \%$, el $76.02 \%$ de los estudiantes han podido presenciar el uso del móvil para hacer plagio. Entonces, una pregunta que surge de manera natural es ¿puede el uso del móvil afectar el proceso académico en los estudiantes de la Universidad Santo Tomás? Al realizar el análisis y de manera sorpresiva, se obtuvo que no existe relación entre el promedio ponderado acumulado de los estudiantes con el tiempo que permanecen conectados a WhatsApp o del uso de las redes sociales como Facebook, Twitter, Instagram, entre otras.

El resultado anterior también fue obtenido por Cabanillas (2018), el cual, también investigó sobre el uso del celular y el rendimiento académico en estudiantes. El trabajo realizado por Cabanillas (2018), tuvo como objetivo principal, describir la relación entre el smartphone y el rendimiento académico. Concluyendo que, aún teniendo un uso excesivo del dispositivo móvil los estudiantes presentan un buen promedio. No obstante, aunque no afecte el promedio el uso del celular en horarios de clase, ocasiona la desatención, interviniendo negativamente en el proceso de aprendizaje, lo cual conlleva a que los estudiantes no participen activamente en las actividades académicas y que haya un desinterés por la clase. Sin embargo, los resultados encontrados en el estudio antes mencionado no son una regla de oro, ni se pueden generalizar para los estudiantes de las demás universidades puesto que, en el estudio realizado con los estudiantes de la Universidad Santo Tomás, se encontró que no solo se utiliza el dispositivo móvil para actividades de ocio, sino también, que aproximadamente $70 \%$ de los estudiantes utilizan frecuentemente o siempre el dispositivo para actividades propias de la carrera, como por ejemplo, investigaciones en internet, como calculadoras en los distintos programas, como tabulador de texto, inclusive, para realizar exámenes.

Esto último nos hace ver desde otra perspectiva el uso del dispositivo móvil, mos- 
trando una cara en donde ciertamente se ven beneficios, utilidad y progreso en el aprendizaje, ya que es evidente el provecho que se le puede sacar a estos dispositivos haciendo un buen uso de ellos, un ejemplo claro en el que se puede afirmar este hecho es un estudio realizado por Jiménez (2017) el cual tuvo resultado positivos al analizar el uso de una aplicación móvil en una población adulta con analfabetismo, obteniendo como resultado que el aprovechamiento de los recursos tecnológicos da frutos, pues facilita y disminuye el tiempo de aprendizaje de la lectura, entre otros beneficios como generación y perfeccionamiento de habilidades. Todas estas herramientas que pueden brindarnos los dispositivos móviles deben y actualmente son aprovechadas tanto por las universidades como los estudiantes.

Por otro lado, un aspecto importante que se quiso indagar en este trabajo está relacionado con la influencia que tiene el dispositivo móvil en la vida de los estudiantes. Para abordar este tema, una de las preguntas más llamativas y con interesantes resultados que se realizó en esta investigación fue: si creía ser adicto o no al celular. Esta pregunta apuntaba a evaluar la dependencia que sentían los estudiantes con el uso continuo del dispositivo móvil. Encontrando que el $43 \%$ de la población estudiantil se considera adicta al celular, dicho esto, se analizó la variable por sexo obteniendo una proporción más alta de mujeres $(23,4 \%)$ que sienten ser adictas al celular con relación a los hombres (19,6\%). El anterior resultado no es sorpresivo puesto que, Ruiz-Palmero, Sánchez-Rodríguez y Trujillo-Torres (2016) afirman que el uso excesivo de teléfonos celulares inteligentes genera problemas de adicción y afecta la vida personal, siendo las mujeres más propensas a tener dependencia que los hombres a los teléfonos móviles. Adicional a lo anterior, las mujeres manifiestan mayor inquietud ante la situación de no disponer de teléfono móvil pues se encontró que $21,07 \%$ de mujeres afirman tener angustian frente a un $18,19 \%$ de hombres.

Otro hito que se quiso abordar en este artículo es que el tener angustia al no poder hacer uso del dispositivo móvil tenía relación con la adicción a dicho dispositivo, pues la angustia por abstinencia resulta ser un síntoma de la gran mayoría de adicciones. Observando que un $60 \%$ de estudiantes de la universidad vive esto, se decide probar esta hipótesis por medio de una prueba de independencia y efectivamente, con una confianza del $95 \%$ el resultado arroja que se relacionan, es decir, que, si una persona pasa angustia al no poder hacer uso de su dispositivo móvil, esta podría tener indicios de adicción.

Otros resultados interesantes que surgieron de esta investigación son mostrados en la Tabla 3 en donde se observa, particularmente, uso del tiempo en redes sociales, utilización de las cámaras fotográficas del celular entre otros aspectos discriminados por sexo. 
Uso y abuso del celular por parte de estudiantes de la Universidad Santo Tomás, una perspectiva estadística41

Tabla 6: Tiempo de uso de aplicaciones desde el dispositivo móvil para hombres y mujeres

\begin{tabular}{|l|lll|lll|}
\hline & \multicolumn{3}{|c|}{ Horas en cámara } & \multicolumn{3}{c|}{ Horas en Whatssap } \\
\hline & Femenino & Masculino & Total & Femenino & Masculino & Total \\
No utilizo & $7,7 \%$ & $19,1 \%$ & $9,1 \%$ & $0,53 \%$ & $0,36 \%$ & $32,44 \%$ \\
Menor a 2 & $33,2 \%$ & $28,3 \%$ & $2,0 \%$ & $7,13 \%$ & $16,76 \%$ & $25,67 \%$ \\
$\mathbf{2}-\mathbf{4}$ & $6,2 \%$ & $2,9 \%$ & $0,7 \%$ & $17,11 \%$ & $15,33 \%$ & $17,11 \%$ \\
$\mathbf{4}-\mathbf{6}$ & $1,6 \%$ & $0,4 \%$ & $61,5 \%$ & $12,83 \%$ & $12,83 \%$ & $23,89 \%$ \\
Más de 6 & $0,5 \%$ & $0,2 \%$ & $26,7 \%$ & $11,59 \%$ & $5,53 \%$ & $0,89 \%$ \\
Total & $49,2 \%$ & $50,8 \%$ & $100 \%$ & $49,2 \%$ & $50,8 \%$ & $100 \%$ \\
\hline & \multicolumn{3}{|c|}{ Horas en redes sociales } & \multicolumn{3}{c|}{ Horas en llamadas } \\
\hline & Femenino & Masculino & Total & Femenino & Masculino & Total \\
No utilizo & $0,53 \%$ & $1,43 \%$ & $38,7 \%$ & $6,24 \%$ & $8,02 \%$ & $13,37 \%$ \\
Menor a 2 & $7,84 \%$ & $12,83 \%$ & $29,6 \%$ & $30,66 \%$ & $34,05 \%$ & $5,17 \%$ \\
$\mathbf{2 - 4}$ & $18,72 \%$ & $19,96 \%$ & $9,1 \%$ & $6,95 \%$ & $6,42 \%$ & $2,50 \%$ \\
$\mathbf{4 - \mathbf { 6 }}$ & $16,04 \%$ & $13,55 \%$ & $20,7 \%$ & $3,21 \%$ & $1,96 \%$ & $64,71 \%$ \\
Más de 6 & $6,06 \%$ & $3,03 \%$ & $2 \%$ & $2,14 \%$ & $0,36 \%$ & $14,26 \%$ \\
Total & $49,2 \%$ & $50,8 \%$ & $100 \%$ & $49,2 \%$ & $50,8 \%$ & $100 \%$ \\
\hline
\end{tabular}

Se encontró que en cuanto al tiempo del uso de la cámara solo el $8.4 \%$ del $49.2 \%$ de la población femenina lo utiliza más de 2 horas, pues prefieren gastar su tiempo en otras actividades como escuchar música, juegos, llamadas o el uso de WhatsApp, donde en promedio el $30 \%$ pasa de 2 a 6 horas diarias. El anterior resultado no es sorpresivo puesto que WhatsApp es el medio de comunicación más popular entre las jóvenes junto con las redes sociales como Facebook, Twitter e Instagram, de las cuales el $35 \%$ gastan las mismas horas diarias en estas aplicaciones. Una explicación al anterior resultado puede deberse a la necesidad de estar conectadas en el mundo digital donde pueden publicar aspectos de su vida o consultar la vida de los demás pero que como hemos dicho anteriormente, esto ha generado problemas de convivencia, inseguridad y hasta narcisismo.

Finalmente, en el género masculino se observa que en promedio el $34 \%$ dedica de 2 a 6 horas a redes sociales habiendo equivalencia con el porcentaje de mujeres, esto quiere decir que este tipo de aplicaciones ha afectado tanto a hombres como mujeres, pues estas tienen la habilidad de ajustarse a múltiples públicos, sus diversos contenidos y nuevas tendencias de redes sociales resultan ser muy llamativas y no discrimina género. esto mismo ocurre en cuanto al tiempo de uso del WhatsApp, no habiendo una diferencia significativa con las mujeres el $28 \%$ dedica entre 2 a 6 horas diarias pues al parecer los ambos sexos han adquirido la misma necesidad de estar en constante comunicación. Ahora bien, en ambos géneros se observa un bajo uso del celular para llamadas siendo el $31 \%$ para mujeres y $35 \%$ para hombres que lo utilizan menos de 2 horas. 


\section{Conclusiones}

Del estudio se obtuvo un primer acercamiento a la caracterización del uso del dispositivo móvil, donde, si bien el uso que se le da en gran parte es dedicado a actividades de ocio y comunicación como WhatsApp, redes sociales y otras aplicaciones, también lo es para actividades propias de la carrera, actualmente este uso es limitado y es por esta razón que al identificar las necesidades de esta nueva generación, será más factible establecer planes de formación académica para los estudiantes, que les permita desarrollar habilidades y que facilite el aprendizaje en todas las áreas del conocimiento, es aquí donde radica la importancia de que los estudiantes aprendan a tener un buen manejo del dispositivo móvil, reduciendo la desatención y desinterés por la clase, y así mismo el riesgo de causas para inicios a otros problemas más graves.

Entre otros resultados se destacó que siete de cada diez estudiantes han evidenciado el uso del móvil para realizar plagio en clase, esto es un ejemplo de mal manejo que han dedicado al dispositivo móvil, y dado que es una cantidad significativa, es pertinente generar estrategias para el control de esta problemática, después de todo el objetivo de caracterizar el uso, es deslumbrar nuevos escenarios en los que probablemente sea necesario crear conciencia, como que el $47 \%$ de la población estudiantil ocasionalmente discute con familiares, amigos u otros por el tiempo que pasa en el celular o que la mayoría de estos lo primero que hacen a levantarse y lo último al acostarse es revisar el celular.

Un resultado sustancial en este estudio es que el uso prolongado del dispositivo móvil ha generado ciertas dependencias y adicciones en más del $40 \%$ de la población estudiantil, así mismo los estudiantes dicen tener sentimientos de angustia al no poder hacer uso del dispositivo móvil. Estos resultados son alarmantes pero discutibles, pues al no ser este un estudio dirigido a probar adicción, no se tienen resultados concluyentes de este, sin embargo, se espera que incite a la creación de nuevos estudios enfocados y especializados a este fenómeno emergente, pues sin duda es algo que merece ser atendido.

Recibido: 07/01/2020

Aceptado: 17/05/2020 


\section{Referencias}

Agar, J. (2013), Constant touch: A global history of the mobile phone, Icon Books Ltd.

Arias, M., Ávila, C., Enciso, L., Garzón, J., Matías, S., Peralta, L. \& Vega, D. (2011), 'Influencia de los smartphones en los estilos de vida de los jóvenes universitarios y jóvenes ejecutivos en la ciudad de bogotá', Universidad Jorge Tadeo Lozano .

Bringué, X. \& Sádaba-Chalezquer, C. (2010), ‘Niños y adolescentes españoles ante las pantallas: rasgos configuradores de una generación interactiva', $C E E$ Participación Educativa 15, 86-104.

Cabanillas, A. L. E. (2018), 'Uso del celular y rendimiento académico en estudiantes de la escuela profesional de derecho, universidad nacional pedro ruiz gallo lambayeque', Repositorio digital institucional Universidad César Vallejo . *https://hdl.handle.net/20.500.12692/25217

Cáceres, J. (2018), 'Análisis de la telefonía móvil en la clase social baja en colombia', Universidad Libre de Colombia .

*http://hdl.handle.net/10901/15930

DANE (2017), Encuesta nacional de calidad de vida - ecv 2016, Technical report, DANE.

*https://www.dane.gov.co/files/investigaciones/condiciones_vida/calidad_vi da/Boletin_Tecnico_ECV_2016.pdf

De Morentin, J. I. M., Cortés, A., Medrano, C. \& Apodaca, P. (2014), 'Internet use and parental mediation: A cross-cultural study', Computers \&6 Education 70, 212-221.

*https://doi.org/10.1016/j.compedu.2013.07.036

Estupiñán, J. C. (2008), 'Influencia de la telefonía móvil en los cambios culturales y sociales de los jóvenes de la universidad javeriana en bogotá - colombia : promoción 2003-2007', Pontificia Universidad Javeriana .

*http://hdl.handle.net/10554/9068

Graner, C., Beranuy, M., Sánchez, X., Chamarro, A. \& Castellana, M. (2007), ¿¿qué uso hacen los jóvenes y adolescentes de internet y del móvil?', Comunicación e Xuventude pp. 71-90.

*https://dialnet.unirioja.es/servlet/articulo?codigo $=2648887$

Gutiérrez, H. A. (2016), Estrategias de muestreo diseño de encuestas y estimación de parámetros, Ediciones de la $\mathrm{U}$.

Gómez-Ramirez, I., Valencia-Arias, A. \& Duque, L. (2019), 'Approach to mlearning acceptance among university students: An integrated model of tpb and tam', International Review of Research in Open and Distributed Learning 
44Julian Enrique Castellanos Rosas, Luz Adriana Rodriguez Padilla \& Cristian Fernando Téllez Piñerez

20(3).

*https://doi.org/10.19173/irrodl.v20i4.4061

Jiménez-García, M. \& Martínez-Ortega, M. d. 1. (2017), 'El uso de una aplicación móvil en la enseñanza de la lectura. información tecnológica', Información tecnológica 28(1), 151-160.

*https://dx.doi.org/10.4067/S0718-07642017000100015

Kish, L. (1965), Survey sampling, New York : John Wiley \& Sons.

Muñiz, J. (2005), 'La validez desde una óptica psicométrica', Acta Comportamentalia: Revista Latina de Análisis de Comportamiento 13(1), 9-20.

*https://www.redalyc.org/articulo.oa?id=274520138002

Muñoz-Miralles, R., Ortega-González, R., López-Morón, M. R., Batalla-Martínez, C., Manresa, J. M., Montellà-Jordana, N., Chamarro, A., Carbonell, X. \& Torán-Monserrat, P. (2016), 'The problematic use of information and communication technologies (ict) in adolescents by the cross sectional joitic study', BMC pediatrics 16, 140.

*https://doi.org/10.1186/s12887-016-0674-y

Organista-Sandoval, J., Serrano-Santoyo, A., McAnally-Salas, L. \& Lavigne, G. (2013), 'Apropiación y usos educativos del celular por estudiantes y docentes universitarios', Revista electrónica de investigación educativa 15(3), 139-156. *http://redie.uabc.mx/vol15no3/contenido-organistaetal.html

Rodríguez, H., Restrepo, L. \& García, G. (2017), 'Habilidades digitales y uso de teléfonos inteligentes (smartphones) en el aprendizaje en la educación superior', Revista Virtual Universidad Católica del Norte (50), 126-142.

*http://revistavirtual.ucn.edu.co/index.php/RevistaUCN/article/view/816/1334

Ruiz-Palmero, J., Sánchez-Rodríguez, J. \& Trujillo-Torres, J. M. (2016), 'Utilización de internet y dependencia a teléfonos móviles en adolescentes', Revista Latinoamericana de Ciencias Sociales, Niñez y Juventud 14(2), 1357-1369.

Särndal, C.-E., Swensson, B. \& Wretman, J. (1992), Model assisted survey sampling, Springer-Verlag New York.

Tellez, C. F., Guerrero, S. \& Pacheco, M. (2014), 'Inferencia bootstrap bayesiana para una proporción en muestreo con probabilidades desiguales', Comunicaciones en Estadística 7(1), 31-48.

*https://doi.org/10.15332/s2027-3355.2014.0001.03

Young, K. S. (2019), 'Internet addiction: The emergence of a new clinical disorder', CyberPsychology $\& 3$ Behavior 1(3), 237-244.

*http://doi.org/10.1089/cpb.1998.1.237 